group 1 (classical catheter) and 90 in group 2 (catheter coated with hydrogel and silver salts). Urine from the patients was tested for 10 days after the insertion of the catheter (reactive dipsticks each day and diagnostic urinalysis every 2 days). The UTI associated with catheterization was defined on the basis of bacterial and cytological criteria $(>100,000$ colony-forming units bacteria $/ \mathrm{mL}$ and $>10$ leucocytes per $\mathrm{mm}^{3}$ ).

Twenty-two UTIs were recorded: 13 in group 1 and 9 in group 2. The cumulative incidence of UTI associated with catheterization was $11.1 \%$ overall: $11.9 \%$ for group 1 and $10 \%$ for group 2 ; the odds ratio was 0.82 (95\% confidence interval, $0.30-2.20$ ); the cumulative incidence for UTI, calculated by the Kaplan-Meier method, was 36.3 overall: 35.2 in group 1 and 36.0 in group 2; the overall incidence density was 19 per 1,000 days of catheterization: 21 in group 1 and 18 in group 2. The differences between the two groups were not significant.

The authors pointed out that there is not enough evidence to conclude that catheters coated with silver salts and hydrogel give greater protection than classical catheters and to recommend widespread use.

FROM: Thibon P, Le Coutour X, Leroyer R, Fabry J. Randomized multi-centre trial of the effects of a catheter coated with hydrogel and silver salts on the incidence of hospitalacquired urinary tract infections. J Hosp Infect 2000;45:117-124.

\section{Studies on the Disinfection of VRE-Contaminated Surfaces}

The incidence of vancomycin-resistant enterococci (VRE) as a nosocomial pathogen has been steadily increasing, and the role of environmental surfaces in the transmission of VRE is incompletely defined. At the recent Association of Practitioners in Infection Control meeting, Rutala, Weber, and Gergen, from the University of North Carolina (UNC) School of Medicine and UNC Hospitals, Chapel Hill, reported a study designed to assess the efficacy of a quaternary ammonium compound (quat) to eliminate VRE from environmental surfaces and to assess the transfer of VRE from environmental surfaces via gloved hands.

Test surfaces were inoculated with a VRE suspension $(\sim 100 \mathrm{bacteria} / \mathrm{sq}$ in). Cleaning methods tested included wiping the inoculated surface with a dry cloth, a cloth immersed in sterile saline, a cloth sprayed with a quat, and a cloth immersed in a quat (drying time 1 minute). Transfer experiments were performed by inoculating a surface as above. The surface was then touched with sterile gloved hands, and, following this contact, the fingertips were pressed onto a blood agar culture plate. Assays were performed using Rodac plates.

Reductions in VRE for dry cloth, saline treated cloth, quat-sprayed cloth, and quat-immersed cloth were $71.2 \%$, $99.5 \%, 95.4 \%$, and $98.1 \%$, respectively. The results were similar for both test surfaces (overbed table, Formica) for both VRE and vancomycin-sensitive enterococci. For transfer experiments, the mean colony count of the inoculated sur- face (overbed table, Formica, linoleum, metal) was 79 VRE/Rodac with 0 VRE transferred.

The authors concluded that all cleaning and disinfecting procedures using a moistened cloth are highly effective in removing or inactivating VRE from contaminated surfaces. They observed no difference in elimination of a vancomycin-susceptible and a vancomycinresistant strain of Enterococcus. Transfer of VRE from a contaminated surface via gloved hands was poor, with a transfer efficiency of less than $1 \%$. These data suggest that currently used disinfection processes likely are highly effective in eliminating VRE. However, surface disinfection must involve contact with all contaminated surfaces. Given the low efficiency of transmission via gloved hands, low-level environmental contamination is not likely to play a major role in VRE transmission for patients on contact isolation.

FROM: Rutala WA, Weber DJ, Gergen MF. Vancomycinresistant Enterococcus sp (VRE): surface disinfection and transmissibility via contaminated surfaces. Presented at the Association for Professionals in Infection Control and Epidemiology, Inc, 27th Annual Conference and International Meeting; June 18-22, 2000; Minneapolis, MN. Abstract.

\section{Effect of Temperature and Soil on Ortho-phthalaldehyde Solution}

In the healthcare setting, high-level disinfectants (HLDs) such as those containing glutaraldehyde are used routinely to prevent the transmission of infections by contaminated medical devices. Ortho-phthalaldehyde, an aromatic dialdehyde, has emerged as an alternative HLD to glutaraldehyde.

Chan-Myers and Roberts from Advanced Sterilization Products, Irvine, California, conducted studies to evaluate the effect of temperature and organic soil (horse serum) concentration on the biocidal activity of ortho-phthalaldehyde solution. The normal use concentration of orthophthalaldehyde is $0.55 \%$ with an exposure time of 12 minutes at $20^{\circ} \mathrm{C}$. Using the suspension test and membrane filtration methodology, $0.3 \%$ or tho-phthalaldehyde solution was tested against Bacillus subtilis spores (American Type Culture Collection 19659 , Rockville, MD) at $20^{\circ} \mathrm{C}$, $25^{\circ} \mathrm{C}, 30^{\circ} \mathrm{C}$, and $35^{\circ} \mathrm{C}$. The same solution with $5 \%, 20 \%$, and $40 \%$ added horse serum was evaluated against Staphylococcus aureus and Pseudomonas aeruginosa at $20^{\circ} \mathrm{C}$. At predetermined time exposures, aliquots were sampled from the test solutions (contaminated with $10^{6}$ cells or spores), neutralized, and processed.

The results showed that the level of biocidal activity was directly related to the study temperature. A 5-log reduction was observed in 3 hours at $35^{\circ} \mathrm{C}$ as compared with 24 hours at $20^{\circ} \mathrm{C}$. For $S$ aureus and $P$ aeruginosa, with an exposure time of 5 minutes or below, a decrease in biocidal activity was observed with increasing horse serum concentration, but there was no difference in efficacy when the exposure time was 10 minutes or longer.

The authors concluded that an increase in organic 